\title{
Association of physical activity and consumption of snacks on weight retention at six months after childbirth
}

\author{
Subha NS Perera ${ }^{1 *}$ \& Chrishantha Abeysena ${ }^{2}$ \\ ${ }^{1}$ Health Promotion Bureau, Ministry of Health, Sri Lanka; ${ }^{2}$ Department of Public Health, Faculty of Medicine, \\ University of Kelaniya, Sri Lanka
}

*Correspondence: perera.subha3@gmail.com

(D)https://orcid.org/0000-0002-5221-0446

DOI: https://doi.org/10.4038/jccpsl.v26i2.8227

Received on 14 September 2019

Accepted on 07 March 2020

\begin{abstract}
Introduction: Postpartum weight gain places a woman at risk of developing overweight and obesity in later life.

Objectives: To determine the factors associated with weight retention at six months after childbirth among mothers who attended child welfare clinics in two medical officer of health (MOH) areas

Methods: This was a descriptive-cross sectional study conducted among all mothers who had been registered before 12 weeks in the antenatal clinics during their last pregnancy and attended field child-welfare clinics for pentavalent vaccination. The sample was 387 recruited consecutively until the required sample was achieved. Validated culturally adopted, pretested questionnaires were used. Logistic regression analysis was performed, and the results expressed as adjusted odds ratio (aOR) and corresponding 95\% confidence interval (CI).

Results: The percentage of mothers who retained weight from a booking visit to six months after childbirth was 67.8\%. Low physical activity ( $\mathrm{aOR}=12.0 ; 95 \% \mathrm{CI}=6.0,23.8)$ and consuming $\geq 1$ snack/day (aOR=2.1; 95\% CI=1.3, 3.4) had positively; and being obese or overweight at booking visit ( $\mathrm{aOR}=0.4 ; 95 \% \mathrm{CI}=0.3,0.7)$ and age $>33$ years $(\mathrm{aOR}=0.5 ; 95 \% \mathrm{CI}=0.3,0.9)$ negatively associated with weight retention at six months after childbirth. No significant associations were observed with parity, education status, income, social class, employment, sleep hours, breastfeeding status, quality of diet and psycho-social stress.
\end{abstract}

Conclusions: Low physical activity and consuming $\geq 1$ snack per day, aged $\leq 33$ years and not being obese or overweight at booking visit were associated with weight retention at six months after childbirth.

Key words: body mass index, obesity, physical activity, postpartum, weight retention 


\section{Introduction}

Women of childbearing age are more prone to be obese than males (1). Excess pregnancy weight gain increases the risk of developing overweight and obesity in later life (2-3) and consequent development of diabetes, hypertension, stroke, arthritis and cancer (4). During the period of pregnancy, there is an increment in mother's weight. Due to loss of extracellular fluid, there is a decrement in weight during the first six weeks of postpartum (3). Thereafter, the weight of mother depends on the individual factors that determine the overall weight. If the postpartum weight is higher than that of pre-pregnancy, it is considered as weight retention (4).

Weight retention after childbirth has been studied in different periods, for example in six weeks (5), three months (6), six months (7), nine months (8) and 18 months (9) after childbirth. One study conducted in USA (2) reported that the prevalence of weight retention at six months after childbirth was $66 \%$, while another study conducted in Taiwan (10) reported it as $70 \%$.

Several studies reported that pre-pregnancy body mass index (BMI) (6, 9-12), education status $(7,10)$, socioeconomic status $(8,10,13)$, breastfeeding status (6-7, 10, 14), physical activity $(9,11,13,15-17)$, dietary habits $(14)$ and psychosocial stress $(9,12)$ were associated with weight retention at six months after childbirth. There were inconsistent results across the studies since the methods used to measure the factors vary.

Up to date, there is no published literature available in Sri Lanka which has addressed factors associated with weight retention at six months after childbirth. The results of this study would lead to plan and implement prevention programs targeting women of childbearing age to reduce overweight and obesity. Thus, the objective of this study was to determine factors associated with weight retention of mothers at six months after childbirth.

\section{Methods}

A clinic-based, descriptive, cross-sectional study was carried out in Biyagama and Kelaniya MOH areas in the district of Gampaha in 2012. All mothers who had been registered before 12 weeks in the antenatal clinic during their last pregnancy and attended field child welfare clinics for pentavalent vaccination with six-month old infants were recruited. Exclusion criteria were mother's age $<18$ years, pregnant at the time of recruitment, on any drug for more than one month and who suffered from chronic diseases.

The sample size was calculated based on 95\% confidence interval (CI) with 5\% degree of accuracy and estimated percentage of mothers who retained weight at six months after childbirth of $66 \%$ (2). After adding $10 \%$ for non-response, the sample required was 380. All eligible mothers were included in the study until the minimum sample size was achieved from all the child welfare clinics in the selected $\mathrm{MOH}$ areas.

Six pre-tested instruments were used for data collection. Those were International Physical Activity Questionnaire (IPAQ) Short form (17-18), 12-item General Health Questionnaire (GHQ-12) (19), Modified Life Events Inventory (MLEI), food frequency questionnaire (FFQ) (16) and a self-administered questionnaire to collect basic demographic data, parity, duration of sleep, breastfeeding, contraceptive details and availability of a helper.

Duration of sleep was measured as total hours of actual sleep for 24 hours (not merely going to bed). Breastfeeding was measured using lactation score (13-14), which combines the duration and exclusivity of breastfeeding and had been used in previous literature. IPAQ Short has been validated and adapted for community-based studies among 15-69-year old adults (15) as well as pregnant mothers (17). The level of physical activities was calculated according to the guidelines (19) based on moderate and vigorous activities, and walking. The GHQ-12 detects potential non-psychotic psychiatric disorders using a scoring system and cut-off point taken as $\geq 3$ for the presence of disorder (18). Psycho-social stress was screened with the 28-item MLEI while presence of $\geq 2$ life events was taken as the presence of psycho-social stress. FFQ measured the quality of diet based on 21 food items categorized into 11 food domains. The usual consumption of each food item was assessed using a 5-point Likert scale. Each response was given a weighted score, and based on a cut-off value, each food domain was categorised as either optimal or failed/ suboptimal consumption (16). Dietary habit was measured with consumption frequency of snacks (14).

Weight retention was defined as the weight gain during the period of the first visit to the clinic and six months after childbirth. The weight measured before 
12 weeks of gestation at an antenatal clinic and at 180 to 240 days after childbirth at a child welfare clinic was taken. Underweight, normal weight, overweight and obesity were defined as BMI of $<18.5 \mathrm{~kg} / \mathrm{m}^{2}$, 18.5$24.9 \mathrm{~kg} / \mathrm{m}^{2}, 25-29.9 \mathrm{~kg} / \mathrm{m}^{2}$ and $\geq 30 \mathrm{~kg} / \mathrm{m}^{2}$, respectively. Social class was defined according to the employment of the head of family (20). Five medical undergraduates were trained as data collectors.

\section{Data analysis}

The Statistical Package for Social Science (SPSS) version 16.0 was used for data analysis. Results were expressed as odds ratio (OR) and 95\% CI. All variables in the bivariate analysis with $\mathrm{p}<0.2$ were eligible for multiple logistic regression model. Analysis was performed using purposeful selection including interaction effects. Hosmer and Lemeshow test for goodness of fit was observed to be satisfactory $\left(\chi^{2}=4.24 ; \mathrm{p}=0.84\right)$.

\section{Results}

Of the 410 eligible women, 14 did not participate giving a non-response rate of 3.3\%. Nine mothers were excluded from the study since they did not meet the inclusion criteria of age $<18$ years and the final study sample was 387.

The mean age of participants was $29.6(\mathrm{SD}=4.3)$ years. The majority were aged $25-29$ years $(n=173$; $44.7 \%)$ and belonged to the Sinhala ethnicity $(n=320$;
$82.5 \%)$; were educated up to grade 6-11 ( $\mathrm{n}=284$; 73.4\%); unemployed ( $n=320 ; 82.7 \%)$; and belonged to social class IV ( $\mathrm{n}=110 ; 28.6 \%$; partially skilled workers). Most of the mothers ( $n=203 ; 52.5 \%)$ had a normal BMI at the booking visit, while $29.2 \%(n=113)$ were overweight and 5.4\% $(n=21)$ were obese. Only $12.9 \%(n=50)$ were underweight.

The weight change ranged from $-17.8 \mathrm{~kg}$ to 28.4 $\mathrm{kg}$ after six months of postpartum. Average weight retention was $2.4 \mathrm{~kg}$ (95\% $\mathrm{CI}=2.0,2.8)$. A total of 263 mothers (67.8\%) had retained weight at six months after childbirth, of whom 30 (11\%) had gained weight more than $5 \mathrm{~kg}$. Only 3.3\% $(\mathrm{n}=13)$ had no weight retention. The weight at six months of postpartum was less than the level of their booking visit in 112 (28.8\%) mothers. Almost $92 \%(n=46)$ of the mothers who were less than $18.5 \mathrm{~kg} / \mathrm{m}^{2}$ of BMI during early pregnancy had gained weight from the first trimester of pregnancy to six months after childbirth. The corresponding figures for normal BMI (18.5-24.9 kg/m²) and overweight/obese $\left(\geq 25.0 \mathrm{~kg} / \mathrm{m}^{2}\right)$ were $70.4 \%(\mathrm{n}=143)$ and $55.2 \%(n=74)$.

The associations between probable factors and weight retention at six months after childbirth are shown in Table 1. Statistically significant relationships were observed between the weight gain from first antenatal visit of the pregnancy to six months after childbirth while mothers of $>33$ year old, being overweight or obese at the booking visit, consumption of $\geq 1$ snack per day and engaging in low physical activity.

Table 1. Unadjusted odds ratios for the association with weight retention at six months after childbirth and independent variables

\begin{tabular}{|c|c|c|c|c|c|}
\hline \multirow[b]{2}{*}{ Exposure variable } & \multicolumn{2}{|c|}{$\begin{array}{c}\text { Weight retention after } \\
\text { childbirth, No. (\%) }\end{array}$} & \multirow[b]{2}{*}{ OR } & \multirow[b]{2}{*}{$95 \% \mathrm{CI}$} & \multirow[b]{2}{*}{ p value } \\
\hline & $\begin{array}{c}\text { Yes } \\
(n=263)\end{array}$ & $\begin{array}{c}\text { No } \\
(n=124)\end{array}$ & & & \\
\hline Maternal age $>33$ years & $216(82.1)$ & $88(71.0)$ & 0.5 & $(0.3,0.9)$ & 0.01 \\
\hline Sinhala ethnicity & $218(82.9)$ & $102(82.9)$ & 1.0 & $(0.6,1.8)$ & 0.9 \\
\hline Educated up to GCE (Ordinary Level) & $196(74.5)$ & $90(72.6)$ & 1.1 & $(0.7,1.8)$ & 0.7 \\
\hline Paid employment & $45(17.1)$ & $22(17.7)$ & 1.0 & $(0.6,1.7)$ & 0.9 \\
\hline
\end{tabular}




$\begin{array}{lccccc}\text { Social class IV/V } & 116(44.3) & 55(44.7) & 1.0 & (0.6,1.5) & 0.9 \\ \text { Income Rs. }<20,000 & 136(51.7) & 56(45.2) & 1.3 & (0.8,1.9) & 0.2 \\ \text { Overweight/obese } & 74(28.1) & 60(48.4) & 0.4 & (0.3,0.6) & <0.001 \\ \text { Parity } \geq 2 & 85(32.3) & 43(34.7) & 0.9 & (0.6,1.4) & 0.6 \\ \text { Use of hormonal contraceptive } & 74(28.2) & 31(25.4) & 1.2 & (0.7,1.9) & 0.6 \\ \text { Availability of a helper } & 187(71.1) & 85(68.5) & 1.1 & (0.7,1.8) & 0.6 \\ \text { Sleep }<8 \text { hours/day } & 166(63.1) & 76(61.3) & 1.1 & (0.7,1.7) & 0.7 \\ \text { Lactation score }<12 & 36(13.7) & 18(14.5) & 0.9 & (0.5,1.7) & 0.9 \\ \text { Low physical activity } & 139(52.9) & 11(8.9) & 11.5 & (5.9,22.4) & <0.001 \\ \text { Consuming } \geq 1 \text { snack per day } & 163(66.0) & 50(44.6) & 2.4 & (1.5,3.8) & <0.001 \\ \text { Consuming a suboptimal diet } & 231(92.9) & 109(91.7) & 1.2 & (0.5,2.3)\end{array}$

$\mathrm{OR}=$ odds ratio; $\mathrm{CI}=$ confidence interval; $\mathrm{GHQ}=$ General Health Questionnaire

Table 2 shows results of the final logistic regression model. A statistically significant association was observed with low physical activity, consumption of $\geq 1$ snack per day, being $\geq 33$ years of age, obese or overweight in their booking visit and weight retention at six months after childbirth.

Table 2. Adjusted odds ratios for the association with weight retention at six months after childbirth and independent variables

\begin{tabular}{lccccc}
\hline Variable & B & $\begin{array}{c}\text { Stranded } \\
\text { Error }\end{array}$ & $\begin{array}{c}\text { OR } \\
\text { Ratio }\end{array}$ & $\begin{array}{c}\text { 95\% CI } \\
\text { for OR }\end{array}$ & p value \\
\hline Low physical activity & 2.48 & 0.35 & 12.0 & $(6.0,23.8)$ & 0.001 \\
Consuming snack/day $\geq 1$ & 0.74 & 0.25 & 2.1 & $(1.3,3.4)$ & 0.004 \\
Obese/overweight & -0.83 & 0.26 & 0.4 & $(0.3,0.7)$ & 0.001 \\
Age $>$ 33 years & -0.69 & 0.30 & 0.5 & $(0.3,0.9)$ & \\
\hline
\end{tabular}

B=beta co-efficient; OR=odds ratio; $\mathrm{CI}=$ confidence interval 


\section{Discussion}

Low physical activity and consuming $\geq 1$ snack/ day had positively and being obese or overweight at booking visit and age $>33$ years were negatively associated with weight retention at six months after childbirth. No other factors related to socio-demography, sleep, pregnancy related factors, psychosocial stress and quality of diet were associated with weight retention.

Low physical activity is likely to increase weight retention. This finding is compatible with six other studies (7, 10, 13, 15, 21-22). Different instruments had been used to measure physical activity in literature. Irrespective of the method used, almost all the studies found inverse association with weight retention at six months after childbirth.

Consumption of $\geq 1$ snack per day was significantly associated with weight retention at six months after childbirth. Our findings are consistent with the results of another study (14). In our sample, $40.7 \%$ of mothers did not have a single snack per day. Across-country comparison of diet is difficult since it differs according to the culture. Our study found no association between exclusive breastfeeding for six months and the weight retention at six months after childbirth. This is in keeping with the findings of another study (7). In contrast, one study (23) found that exclusive breastfeeding was associated with greater postpartum weight retention.

Females who had low/normal BMI at booking visit retained more weight at six months after childbirth compared to the females who were overweight/obese. This finding is compatible with five other studies done in Asia $(10,24)$ and in western countries $(6,11,25)$. In contrast, several studies (12-13) from developed countries have shown that as the pre-pregnant BMI increases, there is an increase in weight retention at six months after childbirth. Gunderson (25) found in a longitudinal study that obese and overweight women tend to retain weight six weeks after childbirth and were heavier at six months than women who were of normal/low BMI before pregnancy. In our study, we recruited study participants from community-based settings, whereas other studies $(11,13,24)$ had recruited them predominantly from hospital settings. Therefore, the selection bias is minimal in our study with findings being more generalizable. We also extracted data on weight at the first antenatal visit from pregnant mothers' register, which has been maintained by the field staff whose procedure for measuring weights and record keeping has been standardized. Butte et al. (26) and Lindsay (27) explained that when the first trimester BMI is low, there is an increase in the rate of weight gain and fat deposition during pregnancy, which is not lost even six months after childbirth.

According to our study, when the age increases $\geq 33$ years, the likelihood of retaining weight at six months after childbirth decreases. However, some studies had found that there is no association between the age at conception and weight retention at six months after childbirth $(2,6)$. Varying results across the studies may be due to age distribution among study participants and the method of measuring weight retention. In adolescents, fat mass and linear mass are increasing throughout the pregnancy, so that in early age, weight retention is more than the older age (26). Further, there was no demonstrable association with the duration of sleep and weight retention at six months after childbirth. However, one study (28) reported that there was an association of fewer sleep hours and postpartum weight retention in six months.

In this study, we found no association between the psych-social stress with weight retention at six months after childbirth. Our results are consistent with another study (10) and in contrast with two other studies $(9,12)$. However, comparisons between these studies are difficult since different tools with varying sensitivity/specificity levels have been used to measure different aspects of psychosocial support /parenting stress in the six months after childbirth. In the Sri Lankan culture, the postpartum period is regarded as a vulnerable period and extended family support is available in most of the time.

There was no significant association of ethnicity and religion with weight retention at six months after childbirth. Studies done in western countries $(7,13,26)$ have constantly found an association between weight retention at six months after childbirth and black women. Confounding factors such as early marriage and pre-pregnant body weight should be considered when assessing association with ethnicity.

In this study, education status was not significantly associated with weight retention at six 
months after childbirth. This is consistent with two study findings with Lyu (10) and Pereira et al. (21). In contrast, another two studies $(9,13)$ found an association between education status and weight retention at six months after childbirth. Across country, comparison is difficult due to lack of uniformity in these classifications.

We found no significant association of income and the social class with the weight retention at six months after childbirth. This finding is consistent with one study (10). In contrast, three studies $(7,9,15)$ reported that weight retention at six months after childbirth was associated with low socio-economic classes. Declaration of income can be incorrect for most of the time while designation of occupations will not provide accurate information. Further classification of income and social class are not consistent across the studies.

There is no relationship observed between the mothers' current employment status and weight retention at six months after childbirth. This is compatible with the findings of three studies $(16,22$, 24), which reported no association.

In this study, no association was found between mother's parity and weight retention at six months after childbirth. This finding was consistent with three other studies $(6,9,29)$. In contrast, two studies $(23,28)$ found that there was more weight gain in primiparity than in multi-parity. Inconsistencies among studies may be due to uncontrolled confounding factors like educational status, age, lactation status, maternal employment and the accuracy of reporting parity (30).

One of the limitations of our study was that most of the instruments used were supposed to measure the exposures during the previous 1-2 weeks. Our study has been conducted in community-based child welfare clinic settings in two $\mathrm{MOH}$ areas. Considering the facts that employed mothers and those with good socioeconomic status would underutilize the government service, generalizability of the results should be done with caution. Gestational weight gain which is the most significant established factor was not measured in this study, which would warrant a longitudinal study design. A systematic review reported that gestational weight gain had a positive effect on postpartum weight retention, however there were no consistent conclusions for other factors (1).

\section{Conclusions}

The factors associated with weight retention at six months after childbirth were low physical activity, consuming $\geq 1$ snack/day, being obese or overweight at booking visit and age $>33$ years of the mother. Thus, educating primary care staff regarding the weight gain six months after childbirth is recommended. Mothers should be educated regarding healthy snacks, for example, vegetables, fruits while physical activity should be encouraged among mothers after childbirth. Greater emphasis should be placed on optimizing BMI before pregnancy.

\section{Public Health Implications}

Weight retention six months after childbirth is more prevalent. Those who engage in lower levels of physical activity and frequent consumption of snacks during the postpartum period and are not overweight or obese before pregnancy are more prone to retain weight.

\section{Author Declarations}

Competing interests: The authors declare that they have no competing interests.

Ethics approval and consent to participate: Informed written consent was taken from individual participants prior to data collection and ethical clearance was granted from the Ethics Review Committee of the Faculty of Medicine, University of Colombo.

Funding: Self-funding

Acknowledgements: We are grateful to the Postgraduate Institute of Medicine, University of Colombo, Deputy Provincial Director of Health Services, Gampaha District and the data collectors for the study.

Author contributions: SP participated in the design of the study, coordinated data collection, performed the statistical analysis and drafted the first version of the manuscript. CA participated in the design of the study, performed the statistical analysis, interpreted the data and helped to draft the manuscript. Both authors read and approved the final manuscript. 


\section{References}

1. Cheng HR, Walker LO, Tseng YF, Lin PC. Postpartum weight retention in women in Asia: a systematic review. Obesity Reviews 2011; 12: 770-780.

2. Roony BL \& Schauderger CW. Excess pregnancy weight gain and long-term obesity one decade later. Obstetrics and Gynecology 2002; 100(2): 245-252.

3. Linne Y, Dye L, Barkeling B, Rössner S. Weight development over time in parous women. The SPAWN study, 15 years follow-up. International Journal of Obesity 2003; 27: 1516-1522.

4. Centers for Disease Control and Prevention. Adult obesity causes \& consequences. Available from: https:/ /www.cdc.gov/obesity/adult/causes.html.

5. Schmitt NM, Nicholson WK, Schmitt J. The association of pregnancy and the development of obesity - results of a systematic review and metaanalysis on the natural history of postpartum weight retention. International Journal of Obesity 2007; 31: 1642-1651.

6. Gunderson EP. Childbearing and obesity in women, weight before, during, and after pregnancy. Obstetrics and Gynaecology Clinics of North America 2009; 36(2): 317-332.

7. Krause KM, Lovelady CA, Peterson BL, Chowdhury $\mathrm{N}$, Ostbye T. Effect of breast-feeding on weight retention at 3 and 6 months postpartum, data from the North Carolina WIC Programme. International Journal of Nutritional Health 2010; 13(12): 2019-26.

8. Walker LO \& Graves JF. Predictors of weight gain at 6 and 18 months after childbirth: a pilot study. Journal of Obstetric, Gynaecologic \& Neonatal Nursing 1996; 25(1): 39-48.

9. Kac G, D’Aquino Benicio MH, Valente JG, VelásquezMeléndez G. Postpartum weight retention among women in Rio de Janeiro: a follow-up study. Cadernos de Saude Publica 2003; 19 Suppl 1: S149-161.

10. Pederson P, Baker JL, Henriksen TB, et al. Influence of psychosocial factors on postpartum weight retention. Obesity 2011; 19(3): 639-646.

11. Lyu LC, Lo CC, Chen HF, Wang CY, Liu DM. A prospective study of dietary intakes and influential factors from pregnancy to postpartum on maternal weight retention in Taipei, Taiwan. British Journal of Nutrition 2009; 102(12): 1828-1837.

12. Kim H, Ki YK, Lee S, et al. Evaluation of plasma leptin levels and BMI as predictors of postpartum weight retention. The Indian Journal of Medical Research 2008; 128: 595-600.

13. Hoedjes M, Berks D, Vogel I, et al. Postpartum physical activity after pre-eclampsia. Pregnancy Hypertension 2012; 2(2): 143-151.
14. Krause KM, Lovelady CA, Ostbye T. Predictors of breastfeeding in overweight and obese women, data from Active Mothers Postpartum (AMP). Maternal and Child Health Journal 2011; 15(3): 367-375.

15. Vernon MM, Hyman DY, Looney SW. Maternal stress, physical activity, and body mass index during new mothers' first year postpartum. Women Health 2010; 50(6): 544-562.

16. Arambepola C. Abdominal obesity and its association with selected risk factors of coronary heart disease in an adult population in the district of Colombo. MD Thesis (Community Medicine). Colombo: Post Graduate Institute of Medicine, 2004.

17. Sanda B, Vistad I, Haakstad LAH, et al. Reliability and concurrent validity of the International Physical Activity Questionnaire short form among pregnant women. BMC Sports Science, Medicine and Rehabilitation 2017; 9: 7.

18. IPAQ group. Guidelines for Data Processing and Analysis of the International Physical Activity Questionnaire (IPAQ) - Short and Long Forms, 2005. Available from: www.ipaq.ki.se

19. Abeysena C, Jaywardena P, Peiris U. Validation of the Sinhala version of the 12-item General Health Questionnaire. Journal of the Postgraduate Institute of Medicine 2014; 1(1): E8:1-E8:7.

20. Barker DJP, Hall AJ. Practical Epidemiology. 2nd ed. UK: Churchill Livingstone. 1991, 23-5p.

21. Pereira MA, Sheryl L, Shiman K, et al. Predictors of change in physical activity during and after pregnancy project viva. American Journal of Preventive Medicine 2007; 32(4): 312-319.

22. Kac G, Pacheco AH, Araújo DM, Rocha CMM, Sousa EB, Coelh NLP. Associated factors to physical activity practice in leisure in postpartum women. Rev Bras Med Esporte 2007; 13(2): 75e-78e.

23. Shao HH, Hwang LC, Huang JP, Hsu HY. Postpartum weight retention risk factors in a Taiwanese cohort study. Obesity Facts 2018; 11: 37-45.

24. Huang TT, Wang HS, Dai FT. Effect of pre-pregnancy body size on postpartum weight retention. Midwifery 2010; 26(2): 222-231.

25. Gunderson EP \& Adams B. Epidemiology of gestational weight gain and body weight changes after pregnancy. Epidemiologic Reviews 2000; 22(2): 261-274.

26. Butte NF, Ellis KJ, Wong WW, Hopkinson, Smith B. Composition of gestational weight gain impacts maternal fat retention and infant birth weight. American Journal of Obstetrics \& Gynecology 2003; 189(5): 1423-1432. 
27. Lindsay CA, Huston L, Amini SB, Catalano PM. Changes in the relationship between body mass index and percent body fat in pregnancy. Obstetrics \& Gynecology 1997; 89(3): 377-382.

28. Gunderson EP, Rifas-Shiman SL, Oken E, et al. Association of fewer hours of sleep at 6 months postpartum with substantial weight retention at 1 year postpartum. American Journal of Epidemiology 2008; 167(2): 178-187.
29. Hill B, McPhie S, Skouteris H. The role of parity in gestational weight gain and postpartum weight retention. Women's Health Issues 2016; 26(1): 123129.

30. Hill B, Bergmeier $\mathrm{H}$, McPhie S, et al. Is parity a risk factor for excessive weight gain during pregnancy and postpartum weight retention? A systematic review and meta analysis. Obesity Reviews 2017; 18(7): 755-764. 